\title{
Violence ou douceur. Les normes éducatives dans les sociétés grecque et romaine
}

Violence or gentleness. Educational norms in Greek and Roman societies Mit harter oder mit sanfter Hand? Erziehungsprinzipien in der griechischen und römischen Gesellschaft

Violencia o dulzura. Las normas educativas en las sociedades griegas y romanas

\section{Bernard Legras}

\section{OpenEdition}

Journals

Édition électronique

URL : https://journals.openedition.org/histoire-education/2018

DOI : 10.4000/histoire-education.2018

ISSN : 2102-5452

Éditeur

ENS Éditions

Édition imprimée

Date de publication : 1 avril 2008

Pagination : 11-34

ISBN : 978-2-7342-1117-4

ISSN : 0221-6280

\section{Référence électronique}

Bernard Legras, «Violence ou douceur. Les normes éducatives dans les sociétés grecque et romaine », Histoire de l'éducation [En ligne], 118 | 2008, mis en ligne le 01 janvier 2013, consulté le 20 mai 2021. URL : http://journals.openedition.org/histoire-education/2018 ; DOI : https://doi.org/ 10.4000/histoire-education.2018 


\title{
Violence ou douceur
}

\section{Les normes éducatives dans les sociétés grecque et romaine}

\author{
Bernard LEGRAS
}

\begin{abstract}
À la mémoire de Jean-Pierre Vernant ${ }^{1}$
La violence était omniprésente dans le cadre des institutions éducatives du monde antique grec et latin ${ }^{2}$. Henri-Irénée Marrou l'avait déjà souligné, en rappelant que le témoignage de Montaigne sur les écoles de son époque s'appliquait sans conteste aux réalités scolaires de l'Antiquité classique ${ }^{3}$ : "Vous n'oyez que criz et d'enfants suppliciez, et de maistres enyvrez en leur cholere. Quelle maniere pour esveiller l'appetit envers leur leçon, à ces tendres ames et craintives, de les y guider d'une troigne effroyable, les mains armées de fouets ? Inique et pernicieuse forme $»^{4}$. Ce témoignage sonne comme un écho de l'aveu
\end{abstract}

1 Cet article préparé avant le décès du philosophe, helléniste et humaniste, est un hommage à l'auteur d'une œuvre qui comporte une riche réflexion sur la violence dans l'éducation grecque : $c f$. "L'identité du jeune Spartiate ", Mètis. Revue d'anthropologie du monde grec, II, 2 (1987), p. 269-300, repris dans L'individu, la mort, l'amour. Soi-même et l'autre en Grèce ancienne, Paris, Gallimard, 1996 (coll. FolioHistoire), p. 173-209.

2 Sur le thème de la violence dans l'Antiquité grecque et romaine, $c f$. Jean-Marie Bertrand (dir.), La violence dans les mondes grec et romain, Actes du colloque international, Paris, 2-4 mai 2002, Paris, Publications de la Sorbonne, 2005.

3 Henri-Irénée Marrou, Histoire de l'éducation dans l'Antiquité, 6e éd., Paris, Seuil, 1965, p. 397. Sur l'éducation antique, voir aussi Stanley F. Bonner, Education in ancient Rome. From the elder Cato to the younger Pliny, Londres, Methuen, 1977 ; Rosella Frasca, Educazione e formazione a Roma, Bari, Dedalo, 1996 ; Bernard Legras, Éducation et culture dans le monde grec (VIII siède av. J.-C.-IVe siècle ap. J.-C.), Paris, Armand Colin, 2002, en particulier p. 20,70, 95 et 116. Le présent article ne reprend pas le dossier de la pédérastie grecque dans sa dimension pédagogique. La violence est normalement exclue des rapports entre l'éraste (l'amant) et l'éromène (l'aimé) : $c f$. Eva Cantarella, Selon la nature, l'usage et la loi : la bisexualité dans le monde antique, trad. fr. Paris, La Découverte, 1991 (éd. italienne, Rome, 1988).

4 Montaigne, Essais 1, 26, Paris, Gallimard, 2007 (Bibliothèque de la Pléiade), p. 165. 
du traumatisme vécu par saint Augustin dans son école latine du IV siècle de notre ère : "Qui ne serait horrifié, qui ne préférerait la mort, si on lui offrait de subir soit la mort, soit à nouveau l'enfance ? Elle qui fait commencer la vie non par des rires, mais par des pleurs, elle annonce en quelque sorte, et sans le savoir, dans quels maux cette vie vient d'entrer " (La Cité de Dieu XXI, 14) ${ }^{5}$. Huit siècles plus tôt, au IVe siècle avant J.-C., Aristote avait énoncé les principes de l'éducation grecque (paideia) en mettant l'accent sur les efforts qu'elle imposait : "Ainsi donc, que le jeu ne doive pas être le but de l'éducation, c'est bien évident : on ne s'amuse pas en apprenant : la douleur est compagne de l'étude " (Politique VIII, 5, 4).

Les sources antiques, littéraires, épigraphiques, papyrologiques et iconographiques, attestent amplement la présence d'une violence qui s'exerçait à la fois contre les usagers de l'école et du gymnase, entre ces usagers, et contre les maîtres. Il faudra distinguer ici la violence institutionnelle autorisée par la loi ou la coutume et la violence réprimée, s'interroger sur les formes et l'expression de cette violence, mesurer les évolutions dans le cadre d'une étude que nous conduisons de la naissance de l'école de l'âge classique jusqu'à la fin de l'Antiquité ( $\mathrm{V}^{\mathrm{e}}$ siècle avant J.-C.-336 après). Cette évolution pose en effet la question de l'introduction de la douceur (barus) dans les rapports pédagogiques. Nous développerons cette analyse d'abord à Sparte, puis dans le cadre de deux institutions antiques : d'une part, le gymnase grec, où les garçons et les jeunes gens s'entraînent aux exercices physiques sans doute dès l'époque archaïque (VIII $-\mathrm{VI}^{\mathrm{e}}$ siècles avant J.-C.), mais surtout à l'époque hellénistique (323-31 avant J.-C.) et romaine, et qui est en général une institution d'État ${ }^{6}$; d'autre part, l'école, quand celle-ci apparaît dans le monde grec, au Ve siècle avant J.-C., et dans le monde romain, aux Ve-IVe siècles, et qui est restée - sauf exceptions - une structure privée ${ }^{7}$.

5 Saint Augustin, La Cité de Dieu, sous la direction de Lucien Jerphagnon, trad. par Catherine Salles, Paris, Gallimard, 2000 (Bibliothèque de la Pléiade). Sauf mention contraire, les traductions sont celles publiées par la Collection des universités de France aux éditions Les Belles Lettres, Paris.

6 On rencontre des gymnases privés dans la chôra égyptienne, mais il s'agit là d'une exception. Le gymnase est en règle générale une institution de la cité grecque : $c f$. Bernard Legras, Néotês. Recherches sur les jeunes Grecs dans l'Égypte grecque et romaine, Genève-Paris, Droz, 1999, passim.

7 Sur la distinction entre école et gymnase, Philippe Gauthier, "Notes sur le rôle du gymnase dans les cités hellénistiques ", in Michael Wörrle et Paul Zanker (dir.), Stadtbild und Bürgerbild im Hellenismus, actes du colloque international, Munich, 24-26 juin 1993, Munich, C.H. Beck, 1995, p. 1-10. 


\section{I - L'éducation spartiate : une violence d'État}

L'éducation spartiate, qui porte le nom d'agôgê à l'époque hellénistique et romaine, se caractérise par son aspect collectif, obligatoire, étatique. La violence y est une donnée permanente : "Le fouet accompagne [...], tout au long de sa carrière de jeune, le futur citoyen de Sparte ${ }^{8}$.

\section{1 - La violence contre les nouveau-nés}

L'agôgê commence à l'âge de sept ans. Mais pour accéder à cette éducation fondée sur les classes d'âge, l'enfant spartiate doit d'abord survivre à l'examen qu'il doit subir après sa naissance. Plutarque rapporte dans la Vie consacrée à Lycurgue, le législateur mythique de la cité laconienne (16, 1-2), que les nouveaunés étaient sélectionnés par les Anciens de la tribu, en un lieu appelé leschê, et qu'il revenait au père d'y présenter l'enfant. On éliminait les nouveau-nés " mal venus et difformes ", qui étaient précipités dans le vide depuis un lieu, les Apothètes, situé dans le Mont Taygète. Ces infanticides étaient dictés - selon Plutarque - par un souci eugénique : " (Les Anciens) jugeaient, en effet, qu'il valait mieux pour l'enfant et pour l'État ne pas le laisser vivre, du moment qu'il était mal doué dès la naissance pour la santé et pour la force ". Les nourrissons qui avaient subi avec succès cet examen étaient lavés - toujours selon Plutarque - avec du vin et non de l'eau, car on croyait que le vin pur ferait mourir de convulsions ceux qui étaient sujets "à la maladie et à l'épilepsie ". On a pu mettre en doute la réalité historique de ces pratiques ${ }^{9}$, mais il est possible de les définir aussi comme des survivances de rites primitifs d'agrégation à la communauté. L'un de leurs buts, à l'époque archaïque, aurait été d'éviter l'accroissement de la population et de maintenir égal le nombre d'attributaires des 9000 lots de terre que Lycurgue aurait assignés à chaque citoyen spartiate ${ }^{10}$.

La vie de l'enfant spartiate jusqu'à l'âge de sept ans est ensuite prise en charge par les nourrices, qui doivent leur apprendre " à n'être point difficiles ni délicats sur la nourriture, à ne pas s'effrayer des ténèbres, à ne pas craindre la solitude, à s'abstenir des caprices vulgaires, des larmes et des cris ». L'enfant spartiate doit apprendre très jeune à maîtriser son corps et son esprit.

8 Jean-Pierre Vernant, "L'identité du jeune Spartiate ", art. cit., p. 195.

9 Cf. Marc Huys, "The Spartan Practice of Selective Infanticide and its Parallels in Ancient Utopian Tradition ", Ancient Society, 27 (1996), p. 47-74.

10 Edmond Lévy, Sparte. Histoire politique et sociale jusqu'à la conquête romaine, Paris, Seuil, 2003 , p. 77. 


\section{2 - L'éducation à l'endurance et à l'obéissance}

Le système spartiate d'éducation est fondé sur les classes d'âge. Il concerne les enfants (paides) de 7 à 12 ans, ainsi que les adolescents (paidiskoi ou meirakia) de 12 à 20 ans. L'objectif général est double : former à la discipline de la vie collective et distinguer une élite.

Les enfants (paides) sont placés sous l'autorité d'un magistrat spécialisé choisi pour sa valeur, le pédonome (paidonomos) ; celui-ci est assisté par des "porteurs de fouet " (mastigophoroi), qui sont de jeunes citoyens. Leur rôle est de stimuler, mais aussi de punir les enfants, selon une discipline toute militaire. Ils peuvent les frapper, et aussi les priver de nourriture. Le vocabulaire traduit le fait qu'il s'agisse d'un véritable dressage. L'unité de base qui regroupe les enfants est nommée boua, c'est-à-dire "troupeau de bovins ". Elle est placée sous le commandement d'un bouagos (" bouvier "). Les bouai sont groupées en une agéla (" troupeau "). Un ensemble d'agelai constitue une ila, une "section de soldats ". Le mot agôgê désigne lui-même " la conduite d'un troupeau ". L'instruction consistait à apprendre l'endurance et le désir de vaincre au combat. Les plus âgés des enfants étaient habitués à marcher pieds nus et à jouer nus. Leur tête était rasée. Ils apprenaient aussi, alors, à lire et à écrire.

L'éducation des adolescents est encore plus sévère. Dès l'âge de 12 ans, ou sinon vers 14 ou 15 ans, ils dorment ensemble sur des paillasses qu'ils fabriquent euxmêmes avec des roseaux de l'Eurotas (le fleuve traversant Sparte et la Laconie). Ils apprennent à vivre de manière frugale, à ne pas se laver, à marcher pieds nus, à n'avoir qu'un seul manteau (khitôn) durant l'année, à recevoir peu de nourriture. Le vol est permis, voire encouragé. Le vol d'un renard fournit un exemple éclatant de l'héroïsme spartiate : "Les enfants qui volent se préoccupent tellement de n'être pas pris que l'un d'eux, qui avait dérobé un renardeau et le tenait caché sous son manteau, laissa, dit-on, la bête lui déchirer le ventre avec ses griffes et ses dents et, pour n'être pas découvert, soutint la douleur jusqu'à en mourir " (Plutarque, Vie de Lycurgue 18, 1). Les épreuves organisées à l'autel d'Artémis Orthia pour les éphèbes contribuent à distinguer la future élite citoyenne. Elles sont connues par des sources littéraires, qui diffèrent sur leur caractère. Xénophon voit en elles un jeu (agôn) qui consiste à s'emparer du plus grand nombre de fromages placés sur l'autel, alors que leurs camarades cherchent à les en empêcher en les fouettant (Constitution des Lacédémoniens II, 9). Cicéron (Tusculanes II, 34 ; 46) et Plutarque (Vie de Lycurgue 18 , 2) le décrivent comme une épreuve dont le but est de subir sans broncher une flagellation qui peut aller jusqu'à la mort. L'âge de l'adolescence est aussi celui du 
combat rituel de Platanistas (Pausanias III, 14, 8-10) : le lieu, situé à Sparte même - un îlot auquel on accédait par deux ponts -, était planté de platanes, d'où son nom. Deux groupes d'éphèbes s'y rassemblaient. Après un sacrifice à Achille, ils organisaient un combat de sangliers domestiques. La victoire d'un sanglier était censée préparer celle du groupe qui le faisait combattre. Le lendemain, les deux groupes s'affrontaient à main nue avec une violence extrême (coups de pieds, morsures, arrachage des yeux) en cherchant à se pousser à l'eau.

La cryptie aussi est marquée par la violence. Cette institution a suscité des débats très vifs. Pour les uns, comme Edmond Lévy, elle aurait une fonction essentiellement militaire ${ }^{11}$. Elle comporterait plusieurs stades, marqués par la brutalité : le premier stade consiste à envoyer les jeunes gens dans la montagne, où ils doivent vivre cachés une année entière (d'où leur nom de "cryptes "), sans ravitaillement ni équipement, ceux qui se laissent voir étant châtiés (Scholie à Platon, Lois I, 633b) ; les plus endurants des cryptes, une élite, sont utilisés comme des commandos chargés de missions de courte durée contre les esclaves spartiates, les Hilotes (Plutarque, Vie de Lycurgue 28, 1-7) ; ils ont pour objectif d'éliminer les leaders d'une éventuelle révolte et agissent sur ordre des éphores, les cinq principaux magistrats de Sparte, qui pratiquent donc une politique de terreur destinée à maintenir le pouvoir de la minorité des citoyens spartiates sur la masse des Hilotes. Mais pour Jean Ducat, la cryptie est un élément du système initiatico-pédagogique spartiate : les victimes sont choisies au hasard, le meurtre des Hilotes doit apparaitre comme une mort symbolique, un rituel d'infériorisation, exprimant le mépris que leur portent les Spartiates ${ }^{12}$. Dans l'un et l'autre cas, les jeunes Spartiates, qui ont subi la violence durant leur éducation, deviennent alors les agents de la violence de l'État spartiate contre les Hilotes.

\section{II - Le gymnase grec : violence des usagers, violence des maîtres}

\section{1 - Les usagers du gymnase}

Le gymnase grec comptait différentes catégories d'usagers, des adolescents et des jeunes gens, que les textes grecs font connaître sous les noms d'éphèbes, de

11 Edmond Lévy, " La cryptie et ses contradictions ", Ktèma, 13 (1988), p. 245-252.

12 Jean Ducat, "La cryptie en question ", in Pierre Brulé et Jacques Oulhen (dir.), Hommages à Yvon Garlan. Esclavage, guerre, économie en Grèce ancienne, Rennes, Presses universitaires de Rennes, 1997, p. 43-74, et, du même, " La cryptie en question ", Cahiers du Centre Gustave-Glotz, 8 (1997), p. 9-38. 
néoi, de néaniskoi et de néotéroi. Les enfants (paides), c'est-à-dire les garçons qui ne sont pas encore entrés dans l'adolescence, s'entraînaient normalement dans un établissement d'éducation physique appelé palestre. Mais dans les petites cités qui ne pouvaient édifier les deux bâtiments, les enfants pouvaient fréquenter le gymnase. À l'époque hellénistique, les deux termes se confondent souvent. À cette époque, les gymnases accueillent également l'enseignement intellectuel de niveau " secondaire " et des conférences. Durant l'époque romaine, les gymnases se transforment très souvent en thermes et sont désormais ouverts à tous les publics. Une inscription publiée en 1977, dont Philippe Gauthier et Miltiade B. Hatzopoulos ont assuré un commentaire détaillé en $1999^{13}$, la loi gymnasiarchique de Béroia régissant la vie d'un gymnase à l'époque hellénistique dans une cité de Macédoine (que l'on doit considérer comme un État de droit), permet de préciser le sens exact de cette terminologie. Cette loi a été adoptée au début du II ${ }^{\mathrm{e}}$ siècle avant J.-C. Ses attendus précisent qu'elle a été votée et affichée pour que les jeunes fassent preuve de plus de retenue (aiskhunê) et obéissent mieux à leurs chefs. Le gymnase accueille les " moins de trente ans ", les hommes faits n’y ont pas accès : les éphèbes y ont environ 18-20 ans ; les termes néoi, néaniskoi et néotéroi sont équivalents et désignent les jeunes gens de 20 à 30 ans. Il est ouvert à certaines heures de la journée aux " enfants ", probablement aux 12/14-16/18 ans, c'est-à-dire aux adolescents qui y viennent sous la conduite de leurs maîtres, les pédotribes. L'accès au gymnase grec est fermé aux esclaves, aux infirmes et aux malades, aux prostitués. La loi de Béroia exclut également les affranchis. Les étrangers libres participent dans certaines cités grecques à la vie du gymnase : dans l'Égypte hellénistique, des Égyptiens pouvaient être admis, ce qui leur ouvrait la voie à l'intégration dans la "Communauté des Hellènes " (les Grecs vivant dans le pays depuis la conquête d'Alexandre le Grand en 332 avant J.-C.). Les valeurs enseignées au gymnase s'expriment en grec par des mots qui reviennent dans les très nombreuses inscriptions louant les qualités des usagers : la prestance (euexia), la discipline (eutaxia) et l'endurance (philoponia).

\section{2 - Violences et délits commis au gymnase}

Les violences au gymnase s'exercent dans un cadre légal à l'encontre des usagers qui ne respectent pas les valeurs et le bon ordre de l'institution. Les punitions sont administrées par le chef du gymnase, le gymnasiarque, qui est normalement

13 Philippe Gauthier et Miltiade B. Hatzopoulos, La loi gymnasiarchique de Béroia, Athènes/Paris, diff. de Boccard, 1993 (Melétêmata 16). 
élu, dans les cités grecques, parmi les hommes faits, c'est-à-dire parmi les plus de trente ans, par l'assemblée des citoyens. Les manquements à la loi et au règlement dans un gymnase sont de différents ordres. Les interdits portent sur le respect dû aux maîtres, sur d'éventuels différends entre usagers qui en viendraient à s'affronter physiquement ou à s'insulter, sur l'interdiction de toute tentative de séduction d'un enfant par un jeune homme ou un adulte.

Les maîtres ont pour tâche d'imposer la discipline. Elle porte sur la ponctualité, tout gymnase (comme toute école) ayant des heures d'ouverture et de fermeture. L'heure du début de l'entraînement est donnée par un symbole visuel (un drapeau) et par un signal sonore. Plaute mentionne ainsi, dans les Bacchides (v. 424-425), que celui qui n'est pas arrivé à la palestre " avant le soleil levant (reçoit) du maître du gymnase une bonne correction " (haud mediocris poenas). Les usagers doivent obéir aux ordres. Les maîtres punissent aussi les fraudes dans les concours. Le caractère impératif de ces règles s'explique largement par le fait que la formation du gymnase est une préparation militaire, les élèves et les néoi constituant les futurs cadres ou soldats de l'armée des cités ou des royaumes hellénistiques.

Le gymnase peut aussi connaître des querelles, des conflits, voire des meurtres. Leur nombre est cependant limité, ce qui tendrait à accréditer l'idée que la vigilance des chefs de gymnase fut rarement prise en défaut et que le contrôle social sur l'institution demeura ferme ${ }^{14}$. Plutarque rapporte, dans sa Vie d'Alcibiade $(3,1)$, que celui-ci " tua d'un coup de bâton un de ses serviteurs dans la palestre de Sibyrtios ", mais il ajoute qu'il pourrait s'agir d'une calomnie lancée " par haine " dans les Invectives d'Antiphon. Et la voie de fait en question ne porte pas sur un autre usager du gymnase. Il en va différemment d'une autre affaire, connue grâce à l'orateur athénien Lysias dans le fragment du discours Contre Teisis (Fragment XVII, 2) :

Archippos que voici, juges, était allé s'exercer dans la même palestre que Teisis, l'accusé. Là, une querelle s'éleva entre eux : railleries, altercation, dispute et injures réciproques. Pythéas est amoureux du jeune homme - il faut vous dire toute la vérité - et il est son tuteur, de par le testament du père. Lorsque Teisis lui eut raconté la scène de la palestre, désireux de lui être agréable, de se donner l'air d'un habile homme et d'un malin, Pythéas, comme les événements nous l'ont fait voir et comme des gens bien informés nous l'ont appris, lui suggéra de se réconcilier pour le moment, mais de chercher le moyen de surprendre son adversaire seul quelque part [suit le récit de la machination de Teisis contre Archippos].

$14 C f$. Ph. Gauthier et M. B. Hatzopoulos, La loi gymnasiarchique, op. cit., p. 95 (2) : "Vu le grand nombre de décrets honorifiques qui louent la discipline des éphèbes ou des néoi, les allusions à la désobéissance ou au désordre paraissent plutôt rares ". 
Le jeune Teisis, aimé par Pythéas, suit ici les mauvais conseils de ce tuteur, mais le fragment ne donne pas la cause du conflit qui éclate dans la palestre entre Teisis et Archippos. Les conflits violents entre jeunes gens sont attestés par d'autres sources, même s'ils ne se déroulent pas dans le cadre du gymnase ${ }^{15}$.

Les délits durant les heures de gymnase pouvaient aussi relever de pratiques de séduction homoérotiques n'entrant pas dans le cadre licite. L'interdiction de mêler aux mêmes heures les " enfants " et les usagers plus âgés au gymnase de Béroia est une mesure manifestement destinée à protéger les enfants des manœuvres de séduction des adultes. Mais cette mesure ne doit pas être interprétée comme une interdiction de la pédérastie ou comme une mesure destinée à protéger les adolescents des menées d'amants "indignes ", car de rang social insuffisant ${ }^{16}$. Il s'agit bien plutôt, à Béroia, d'empêcher que les adolescents qui s'entraînent soient perturbés par des adultes. Les sociétés grecques ne condamnent pas la pédérastie, mais contrôlent les conditions de sa pratique ${ }^{17}$. En contraste avec la loi de Béroia, les Athéniens adultes se rendaient dans les palestres pour voir et tenter de séduire les plus beaux adolescents. Platon en donne un témoignage éclatant dans le Lysis, où Hippothalès vient admirer le beau Lysis dès que celui-ci vient à la palestre (203a-205a), les exercices se faisant dans une totale nudité (gymnos signifie " nu "). Plaute note d'ailleurs que d'autres tentations peuvent exister quand il mentionne qu'il convient, à la palestre, de s'exercer "à la course, à la lutte, au javelot, au disque, au pugilat, à la balle, au saut ; non à faire l'amour, et à embrasser " (Bacchides, v. 28-29). La loi de Béroia interdit également de se "mettre nus " quand le gymnase ferme. Le départ des maîtres et surtout l'obscurité naissante peuvent alors favoriser des situations que la morale condamne.

Les gymnasiarques peuvent enfin être pris à partie dans le gymnase. La loi de Béroia mentionne deux délits : les injures et les coups. Elle exige que les témoins,

$15 C f$. Démosthène, Contre Conon 3-6 (voies de fait, coups et outrages entre jeunes gens dans la garnison athénienne de Panakton) ; Wilhelm Dittenberger, Sylloge Inscriptionum Graecarum, $3^{\mathrm{e}}$ éd., Leipzig, 1915-1924, n 1109, 1. 72-95 (injures dans le règlement des Iobacches) ; Étienne Bertrand, Inscriptions grecques d'Égypte et de Nubie au musée du Louvre, Paris, Éditions du CNRS, 1992, nº 4 (tumulte provoqué par les jeunes au sein de l'assemblée de Ptolémaïs, en Haute Égypte). Sur cette dernière affaire, cf. B. Legras, Néotês, op. cit., p. 228-231.

16 Cette hypothèse faite par E. Cantarella, Selon la nature, l’usage et la loi, op. cit., p. 51, est récusée à juste titre par Ph. Gauthier et M. B. Hatzopoulos, La loi gymnasiarchique, op. cit., p. 85.

17 Cf. E. Cantarella, Selon la nature, l'usage et la loi, op. cit., p. 35 sqq. Le principe fondamental chez les Grecs est que l'aimé doit avoir au moins douze ans, qu'il doit cesser d'avoir des relations passives quand le système pileux apparait et que son amant doit contribuer par son savoir et sa position sociale à son éducation. 
c'est-à-dire les jeunes gens, s'interposent. S'ils ne le font pas, ils sont soumis à l'amende. Un autre délit est le refus d'exécuter les ordres.

\section{3 - La sanction des violences et délits}

Cette sanction consistait soit en une amende, soit dans un châtiment corporel. Le gymnasiarque est autorisé à utiliser le fouet dans deux cas de figure : pour mettre fin à un trouble (bagarre entre usagers, agression commise contre lui par un usager, éviction d'un exclu du gymnase) et comme sanction d'un délit commis dans le gymnase. Ce fouet est constitué soit d'un bâton ou d'une baguette souple (rhabdos ou lugos), soit d'un ensemble de bâtons ou baguettes constituant des verges ${ }^{18}$. Plutarque montre ainsi le triumvir Antoine exerçant la charge de gymnasiarque à Athènes en 39/38 avant J.-C. : " laissant derrière lui les insignes de son commandement, il sortait avec les baguettes (rhabdoi) des gymnasiarques, en manteau et en sandales blanches, et il attrapait par le cou les jeunes gens qu'il fallait séparer " (Vie d'Antoine 33, 7) ${ }^{19}$. La nature de la sanction varie suivant les gymnases. L'amende est en général la punition des éphèbes et des jeunes gens. Le fouet et la bastonnade peuvent n'être infligés qu'aux seuls enfants : la loi de Béroia, qui prévoit le fouet (ou "verges " : rhabdos en grec), est très claire sur ce point, mais elle ne précise pas le nombre de coups que reçoit le fautif ; il est probable que ce nombre est de $50 !^{20}$ Les éphèbes athéniens et ceux d'autres cités se voient, en revanche, sanctionnés par ce châtiment. Un bas-relief athénien (IG II ${ }^{2}$ 2111) montre en effet trois magistrats athéniens appelés sophronistes, qui sont des maîtres chargés, aux côtés du cosmète, de former les éphèbes à la sôphrosunè et sont munis d'un bâton flexible, instrument de la punition ${ }^{21}$. À Amorgos, dans la cité d'Aigialè, une inscription (IG XII 7, 515, 1. 45-49) mentionne que le gymnasiarque peut contraindre les éphèbes et les "jeunes " (néoi), lors d'une procession, à le suivre " par tout moyen possible ». Une statue de Délos montrait de même, très

18 Pierre Chantraine, Dictionnaire étymologique de la langue grecque, s.v. "rhabdos " : " baguette, badine "; s.v. "lugos" : " toute branche flexible que l'on peut tresser".

19 Trad. de l'édition des Belles Lettres légèrement modifiée.

20 Ph. Gauthier et M. B. Hatzopoulos, La loi gymnasiarchique, op. cit., p. 68.

21 Chrysis Pélékidis, Histoire de l'éphébie attique des origines à 31 avant Jésus-Christ, Athènes/Paris, diff. de Boccard, 1962, p. 108. Cf. aussi le Cynique Télès (III ${ }^{\mathrm{e}}$ siècle avant J.-C.), Teletis Reliquiae, $2^{\mathrm{e}}$ éd., par Otto Hensen, Tübingen, Mohr, 1909, n V, p. 50 : "Le voici devenu éphèbe : de nouveau, il craint le cosmète, le pédotribe, le maître d'armes, le gymnasiarque. Il est fouetté par eux tous, on le surveille, il est mis sous le joug " (trad. André-Jean Festugière, Deux prédicateurs de l'Antiquité. Télès et Musonius, Paris, Vrin, 1978, p. 40). Il faut donc corriger H.-I. Marrou, Histoire de l'éducation dans l'Antiquité, op. cit., p. 240-242, qui estimait que les punitions corporelles ne concernaient que le premier niveau d'éducation, le niveau "primaire ". 
probablement, un gymnasiarque, Sôsilos, fils de Dôrieus, portant une baguette ou un bâton. La statue du personnage, de taille réelle, est perdue, mais l'inventaire épigraphique du gymnase lui consacre cet article : " une statue d'homme nu, en grandeur naturelle, dans l'exèdre, portant une baguette (rhabdos), consécration des Déliens " (trad. Claude Vial) ${ }^{22}$.

Dans certaines cités, comme Athènes, la violence ultime de l'État, la peine de mort, s'exerce contre les auteurs de vol au gymnase. Démosthène écrit ainsi : " De plus, pour tout vol d'un manteau, d'une fiole d'huile, ou de tout autre objet de minime valeur, commis au Lycée, à l'Académie ou au Kynosarge, comme aussi pour tout vol d'appareil quelconque, dont la valeur dépasse les dix drachmes, commis dans l'un des gymnases ou des ports, la peine fixée par Solon est également la mort " (Contre Timocratès, 114). Cette extrême sévérité doit s'expliquer par le caractère sacré des gymnases, qui sont tous dédiés à des divinités, le plus souvent à Hermès et Hèraklès, et par l'assimilation de ces vols à un sacrilège (hiérosulia) $^{23}$. La sanction peut cependant être plus modeste. À Béroia, le vol au gymnase doit seulement être puni "plus sévèrement qu'un larcin analogue commis dans d'autres lieux et dans d'autres circonstances "24. La loi athénienne sait que certains homicides dans les concours athlétiques, au gymnase ou dans les autres compétitions, sont involontaires ${ }^{25}$. La justice athénienne les juge donc comme tels au tribunal du Delphinion ${ }^{26}$. Il peut arriver, par exemple, qu'un javelot transperce le corps d'un compagnon d'éphébie ou d'un spectateur ! ${ }^{27}$

22 Denis Knoepfler, qui pensait voir un pédotribe dans "La base de Sôsilos à la Synagogue de Délos ", Bulletin de correspondance hellénique, Suppl. I, 1973, p. 233-237, a été corrigé par Claude Vial, " Délos indépendante d'Athènes ", Bulletin de correspondance hellénique, Suppl. X, 1984, p. 241.

Cf. Ph. Gauthier et M. B. Hatzopoulos, La loi gymnasiarchique, op. cit., p. 133.

Ibid., p. 134. versity Press, 1963, p. 73-74.

$27 C f$. Plutarque, Vie de Périclès 36, 5, où est posée la question de la responsabilité du javelot, de l'athlète qui le lance ou des organisateurs de l'épreuve. 


\section{III - L'école grecque et romaine : une pédagogie du châtiment corporel}

L'usage de la violence dans les écoles grecques est attesté à la fois par l'iconographie et par les textes ${ }^{28}$.

\section{1 - L'école grecque : coups de sandale et de bâton, fouet, claques et autres}

La céramique attique montre que la sandale est communément utilisée par les parents pour punir l'enfant (figure 1$)^{29}$.

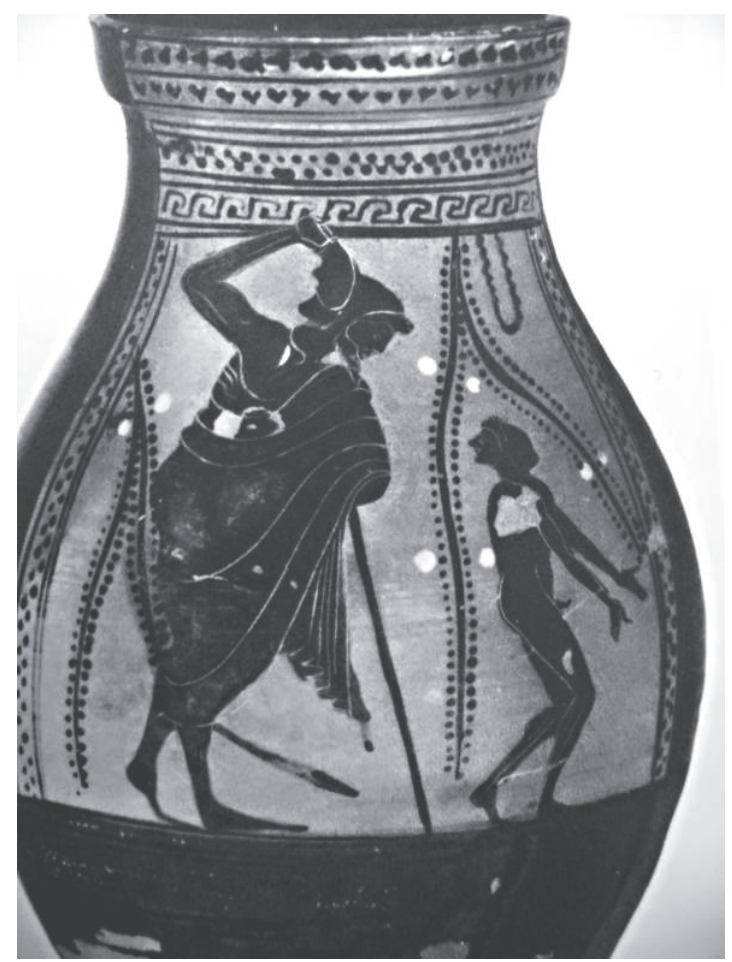

Figure 1 : Les coups de sandale. Oinochoé attique, Berlin, Staatliche Museen 3230, fin du $\mathrm{VI}^{\mathrm{e}}$ siècle av. J.-C.

28 Cf. Alan D. Booth, "Punishment, Discipline and Riot in the Schools of Antiquity ", Échos du monde classique, 17 (1973), p. 107-114 ; Frederick. A.G. Beck, Album of Greek Education. The Greeks at School and at Play, Sydney, Cheiron Press, 1975, p. 43-46 et planches 52-53 ; Stanley F. Bonner, Education in ancient Rome, op. cit., p. 143-145 ; Jean-Pierre Néraudau, Être enfant à Rome, Paris, Les Belles Lettres, 1984, p. 316-323 ; Raffaella Cribiore, Gymnastics of the Mind. Greek Education in Hellenistic and Roman Egypt, Princeton (N.J.), Princeton University Press, 2001, p. 67-73.

29 Berlin, Staatliche Museen, 3230, fin du VI siècle avant J.-C., cf. F. A.G. Beck, Album of Greek Education, op. cit., planche 52, 271, et Bologne, Museo Civico, PU 204, vers 550 avant J.-C., cf. F. A.G. Beck, ibid., planche 52, 272. 
Les coups portés peuvent marquer le corps de l'enfant. Une hydrie attique fait voir un enfant battu (à gauche) avec cinq marques sur le corps, une femme agenouillée suppliant un homme imberbe assis (figure 2$)^{30}$.

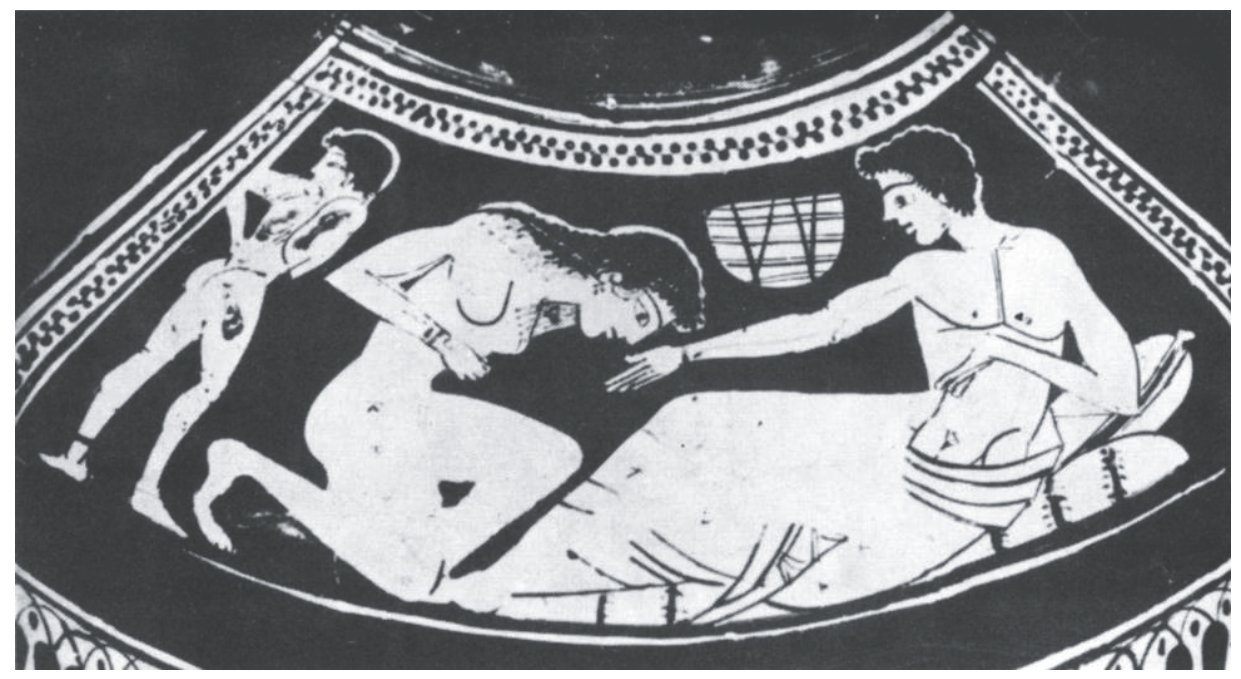

Figure 2 : Les marques de coups. Hydrie attique, Universität Würzburg, Museum Martin von Wagner 530, vers 500 av. J.-C.

À Sparte, les porteurs de fouets (mastigophoroi) assistent le pédonome ${ }^{31}$ (le magistrat chargé de surveiller les enfants spartiates pendant les trois cycles de l'agôgê). À la différence de Sparte, où l'éducation est régie par l'État, l'école athénienne - la seule véritablement connue à l'époque classique - et les écoles hellénistiques sont de statut privé. Les violences physiques infligées aux élèves sont du seul ressort du maitre, sous le contrôle des parents qui lui versent les honoraires. Les corrections infligées par les maîtres d'école sont des coups de sandales, des coups de bâton ${ }^{32}$ et des claques (figure 3$)^{33}$.

30 Universität Würzburg, Museum Martin von Wagner, 530, vers 500 avant J.-C., cf. Beck, ibid., planche 53, 274.

31 Xénophon, République des Lacédémoniens, II, 2.

32 Cf. Bari, Museo Nazionale, R 150, cf. F. A.G. Beck, Album of Greek Education, op. cit., planche 53, 276a.

33 Melbourne, National Gallery of Victoria, Felton Bequest, 1644/4, vers 450 avant J.-C., cf. F. A.G. Beck, ibid., planche 53, 273. 


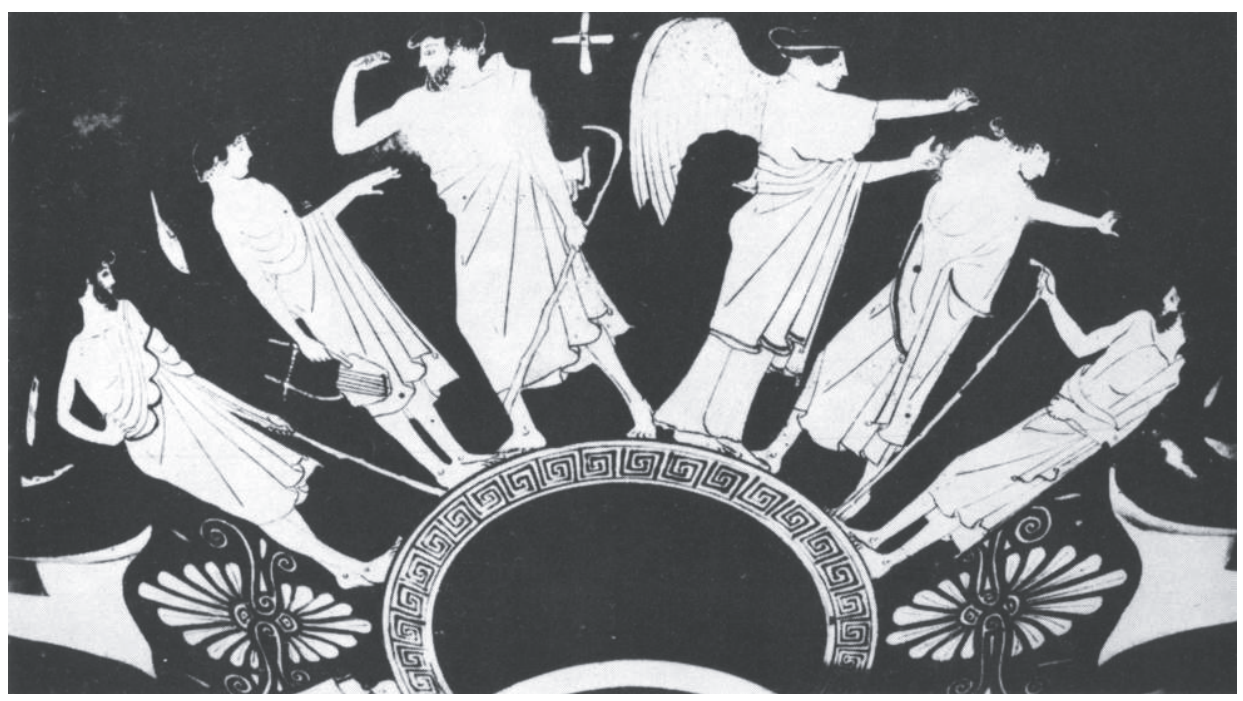

Figure 3 : La claque. Coupe attique, Melbourne, National Gallery of Victoria, Felton Bequest, $1644 / 4$, vers 450 av. J.-C.

Un auteur d'épigrammes du premier siècle avant notre ère, Phanias, atteste que les coups peuvent être administrés à l'école à l'aide de sandales, les deux autres instruments pédagogiques étant la férule (narthêx) - l'" arme de chevet " du maître - et un " épervier " (kikos) bien arrondi, dans lequel il faut sans doute voir une sorte de carcan fait de bois flexible ${ }^{34}$. Le témoignage littéraire le plus saisissant sur l'utilisation du fouet est fourni par Hérondas, un auteur alexandrin de saynètes, les Mimiambes, dans la première moitié du III ${ }^{e}$ siècle avant J.-C., qui décrit une scène de violence scolaire dans le Maitre d'école (Didaskalos, Mimiambe III ${ }^{35}$. Le maître, Lampriskos, menace ainsi l'élève Kottalos, qui est fainéant et fait l'école buissonnière : "Je vais te rendre plus modeste qu'une jeune fille, sage à ne pas lever de terre un fétu, si c'est là ton goût. Où est le cuir dur, ma queue de bœuf dont je bats les réfractaires, ceux qui sont mis aux fers? Qu'on me la mette en mains avant que ma colère éclate " (v. 66-70). Kottalos le supplie alors de ne pas user du fouet le plus rude : "Non, je t'en supplie, Lampriskos, par les Muses, par ton menton et la vie de Koutis ${ }^{36}$, pas le dur! Prends l'autre pour me battre " (v. 71-73). Sa grand-mère décide du nombre de coups, " autant

34 Anthologie Palatine VI, 294.

35 Cf. B. Legras, Néotês, op. cit., p. 88-89.

36 Il s'agirait de la fille du maitre d'école. 
qu'en pourra supporter ta vilaine peau " (v. 79-80) : "Il ne faut pas t'arrêter, Lampriskos, écorche-lui la peau jusqu'au coucher du soleil " (v. 87-88). Il reçoit au total des dizaines de coups. Un gemme conservé à Berlin (figure 4) offre à voir ce châtiment, qui est administré à l'enfant nu, porté sur les épaules d'un assistant ou d'un autre enfant et offrant son dos aux verges ${ }^{37}$.

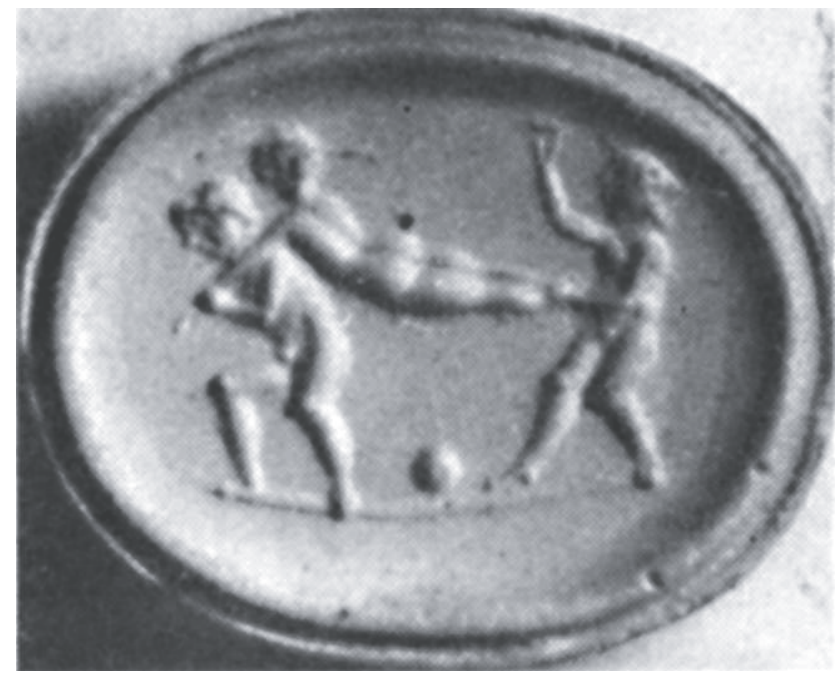

Figure 4 : Le fouet. Gemme, Berlin, Staatliche Museen 6918, époque gréco-romaine.

Ces dernières semblent formées de deux courts bâtons se terminant par une pièce arrondie. Le supplice porte en latin le nom de catomus, terme formé sur l'expression grecque signifiant " sur les épaules " (kat'ômon). Le verbe désignant cette épreuve est en grec katômizein, en latin catomidiare.

La punition corporelle trouve sa justification, selon le sophiste Protagoras, dans le fait que la vertu doit s'acquérir également par la punition, car « l'usage de punir et de frapper ceux qu'on juge coupables est universel et ne se rencontre pas moins qu'ailleurs chez tes compatriotes [c'est-à-dire ceux de Socrate], les Athéniens " (Platon, Protagoras 324 a-c). Elle doit frapper l'enfant, l'homme ou la femme " jusqu'à ce que la correction l'ait amélioré " (ibid., 325a). Platon recommande dans les Lois de traiter avec la plus grande sévérité les enfants qui doivent être punis en cas de faute. Il ne mentionne pas explicitement les punitions corporelles. Mais celles-ci sont implicites, car il met sur le même plan les enfants, le bétail et

37 Staatliche Museen, 6918, cf. F. A.G. Beck, Album of Greek Education, op. cit., planche 53, 275. La date ne peut être exactement déterminée : "époque gréco-romaine ". 
les esclaves : "De tous les animaux, c'est l'enfant le plus difficile à manier ; par l'excellence même de cette source de raison qui est en lui, non encore disciplinée, c'est une bête rusée, astucieuse, la plus insolente de toutes " (Lois 808d).

\section{2 - L'école romaine : la férule et le fouet}

Les châtiments corporels font également partie des pratiques pédagogiques courantes à Rome. Comme dans l'école grecque, la punition était du seul ressort du maître ${ }^{38}$. Elle était aussi pratiquée dans la maison familiale. Le fait qu'elle était également administrée aux esclaves semble cependant avoir constitué un frein pour les familles de l'élite ${ }^{39}$. Le maître était considéré comme "le père " de la " cohue d'enfants " dont il devait surveiller le comportement en classe, dans tous ses aspects ${ }^{40}$.

Les châtiments corporels qui faisaient de l'école un lieu de violence sont devenus un topos dans la littérature latine et grecque. Cette réalité est incontestable. Une peinture placée sous les portiques du forum de Pompéi présente la même scène de flagellation sur le dos d'un enfant (figure 5) que le gemme précédemment cité et la saynète d'Hérondas ${ }^{41}$.

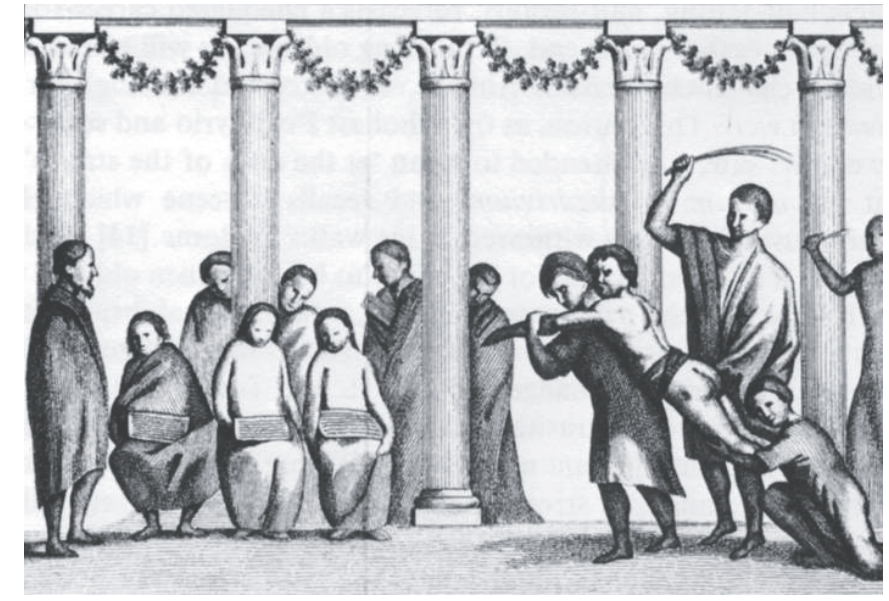

Figure 5 : Le fouet. Peinture du Forum de Pompéi. Naples, Musée archéologique national.

38 Confessions V, 8, 14.

39 Cf. Richard P. Saller, Patriarchy, Property and Death in the Roman Family, Cambridge, Cambridge University Press, 1994, p. 133-153.

40 Juvénal, Satires VII, v. 238-241.

41 Martin P. Nilsson, Die hellenistische Schule, Munich, C.H. Beck, 1955, p. 61 et planche 10 ; Stanley F. Bonner, Education in ancient Rome, op. cit., p. 118 (fig. 11). 
L'enfant est porté par un de ses camarades de classe, alors qu'un second lui tient les pieds. Le fouet semble formé par trois baguettes. Les autres élèves, assis, assistent à la scène. Des élèves de Pompéi ont gravé ces graffitis : " Je reçois des coups ", "reçois des coups " ${ }^{42}$. Plutarque rapporte les propos méprisants de Sertorius à l'encontre de Pompée au moment où ce dernier arrive en Espagne : " Je n'aurais besoin, disait-il en manière de sarcasme, que d'une férule et d'un fouet pour corriger cet enfant " (Vie de Pompée 18, 1). Les maîtres romains pratiquaient deux degrés de punitions en fonction de la gravité de la faute. La peine la plus légère était administrée avec une baguette (ferula) qui tombait sur la paume des mains : Ovide mentionne ainsi les " mains délicates " des enfants sur lesquelles s'abattaient "les verges cruelles " des maîtres ${ }^{43}$; le poète Martial définit la férule comme "le sceptre des pédagogues " (Épigrammes X 62, 10) et comme un élément central de la pédagogie romaine : "Férules (Ferulae). Grandement odieuses aux enfants et chères à leurs maîtres, nous sommes devenues, par le don de Prométhée, un bois illustre ${ }^{44}$.

Le degré supérieur consistait à frapper avec un fouet ou martinet (scutica) formé de lanières de cuir plus ou moins larges. Pline le Jeune rapporte que certains maîtres utilisaient comme fouet la peau séchée d'anguille, qui était assez épaisse (Histoire naturelle IX, 39, 77). Ces châtiments sont des punitions que justifie l'exaspération de maîtres devant un élève aussi inapte aux études que ce " lourdaud, vrai roussin d'Arcadie " dont parle Juvénal dans la Satire VII (v. 158-160).

Certains maîtres étaient réputés pour leur usage immodéré des punitions corporelles. C'est le cas du grammairien L. Orbilius, qui enseigna à Rome à partir de 63 avant J.-C. et qui fut sans doute le maître d'Horace. Selon Suétone ${ }^{45}$, il était " impitoyable, non seulement pour ses adversaires, qu'il ne perdit pas une occasion de déchirer, mais aussi pour ses élèves, comme le laissent entendre Horace lorsqu'il le qualifie de fouettard (plagosus) ${ }^{46}$ et Domitius Marsus lorsqu'il parle de "tous ceux qu'Orbilius a frappés de sa férule (ferula) et de son martinet (scutica)" ". C'est aussi le cas de Rutilus, "qui met sa joie dans le bruit cruel des coups, pour qui le chant des Sirènes n'est pas comparable à la musique des fouets " (Juvénal, Satire XIV, v. 18-20). Ausone encourage son petit-fils à supporter les mauvais

42 Matteo della Corte, Case ed abitanti di Pompei, $2^{\text {e }}$ éd. Pompéi, 1954, p. 112.

43 Ovide, Amours I, 13, 17-18. Cf. aussi Plutarque, Vie de César 61, 3 ; Juvénal, Satires I, v. 15 ; Ausonius, Épitres XXII, 29-30. 
traitements que ne manquera pas de lui infliger son maître "à l'aspect farouche " (horrida forma) (Épîtres XXII, v. 13).

Cette violence exercée par les maîtres avait plusieurs causes. On peut d'abord invoquer le statut social des maîtres, le plus souvent des esclaves et des affranchis qui auraient voulu se venger sur des enfants libres de leur basse condition sociale $^{47}$. Pour certains maîtres de statut libre, l'amertume née d'ambitions déçues a pu jouer : ce serait le cas d'Orbilius, un Romain né en 113 avant J.-C., qui ouvrit son école après avoir dû faire face aux difficultés nées de l'assassinat de ses parents et d'une carrière militaire médiocre. Elle résultait aussi d'une pédagogie qui se voulait coercitive et qui voyait dans la punition corporelle une technique éducative permettant à l'élève de progresser en imposant attention et discipline. Elle était enfin une réponse aux turbulences des élèves.

\section{3 - La violence contre les maîtres et entre élèves}

Les maîtres étaient victimes, de la part de leurs élèves, d'insultes, d'agressions et de règlements de comptes qui dépassaient le simple " chahut ». L. Orbilius écrivit en grec un traité - perdu - probablement intitulé "Le souffre-douleur " (Périalgês) ${ }^{48}$, où il déplorait "le tort que les parents causent aux professeurs par leur indifférence ou leurs prétentions " (Suétone, Grammairiens et rhéteurs IX). Plaute rapporte que certains parents ne dissuadaient pas leurs enfants de s'en prendre à leur maître, en l'occurrence un précepteur privé (Bacchides, v. 440-448) :

Mais aujourd'hui, un marmot qui n'a pas sept ans, si on le touche du bout des doigts, aussitôt il vous fend la tête de son précepteur avec sa tablette. Va-t-on réclamer auprès du père, le père ne manque pas de dire à son garçon : "Tu es bien de notre sang, et tant que tu sauras te défendre contre les injures, tu le resteras". Et l'on menace le précepteur : "Dis donc, vieil imbécile, ne t'avise pas de toucher à cet enfant, parce qu'il a montré du cœur ». Le précepteur ainsi bafoué s'en va, la tête entortillée dans un linge huilé, comme une lanterne. Et le tribunal se sépare sur cette belle sentence. Comment un précepteur peut-il garder quelque autorité, s'il est lui-même le premier battu?

Rufus était traité par ses élèves de "Cicéron allobroge " ${ }^{9}$ : l'insulte était raffinée ${ }^{50}$, mais cruelle, car elle signifiait que le maître s'exprimait comme un

47 J.-P. Néraudau, Être enfant à Rome, op. cit., p. 317.

48 Sur ce titre, voir Marie-Claude Vacher (dir.), Suétone. Grammairiens et rhéteurs, Paris, Les Belles Lettres, 1996, p. 95-96.

49 Juvénal, Satires VII, v. 214.

$50 C f$. le rôle des Allobroges dans la conjuration de Catilina : Salluste, La conjuration de Catilina. 
provincial, les Allobroges étant des pérégrins (c'est-à-dire des non-Romains) de la Gaule Narbonnaise (du Dauphiné et de la Savoie actuels). Des élèves de Pompéi s'en prenaient à leur maître avec des insultes à connotation sexuelle ${ }^{51}$. Libanios mentionne les menaces verbales et physiques d'un étudiant qui avait dilapidé les honoraires confiés par son père pour le maître : " Il en dépense une partie à boire, une autre aux dés, et le restant pour satisfaire un sexe quelquefois plus hardi que la loi ne le permet. Après quoi, rappelant à lui toute son insolence, il entre d'un bond dans la salle, il crie, il menace, il regarde tout le monde comme de l'ordure, et prétend faire accepter son entrée comme seul salaire " (Discours III, 6). Saint Augustin déplore la licence " sans frein " des étudiants de Carthage, qui " commettent mille insolences, avec une stupidité incroyable, que la loi (lex) devrait punir, s'il n'y avait la tradition (consuetudo) pour les protéger " (Confessions V, 8, 14).

La violence potentielle des enfants pouvait être utilisée pour des raisons politiques ou religieuses ${ }^{52}$. Tite-Live $(V, 27)$ rapporte l'histoire du maître d'école de Faléries, alors en guerre contre Rome, qui voulut livrer aux Romains les enfants de cette ville qui lui avaient été confiés : Camille refusa de faire de ces enfants des otages et leur livra le traitre ; ceux-ci le raccompagnèrent à Faléries, dénudé, les mains liées dans le dos, en le frappant. L'empereur Caligula exerça sa cruauté contre un flatteur qui avait offert sa vie pour le rétablissement de l'empereur malade, mais qui tardait à réaliser sa promesse après sa guérison : le malheureux fut "livré aux enfants ", qui le menèrent à travers la ville, " couronné d'herbe sacrée et couvert de bandelettes ", pour le jeter finalement " du haut du mur "53. La scène de violence la plus intense se lit sous la plume de Prudence à propos du martyre de saint Cassien. Prudence la situe naturellement dans un contexte de persécution contre les chrétiens, mais insiste sur le caractère de revanche des élèves contre leur maître :

Cassien avait dirigé une école ; entouré d'un grand nombre d'élèves, il avait occupé une chaire de maitre d'écriture [...]. Son enseignement avait paru quelquefois sévère et dur à ses jeunes auditeurs, et avait fait naître chez eux de la rancune et de la crainte : le maître, en effet, est toujours pénible pour le jeune homme qui apprend, et les enfants ne trouvent jamais l'étude agréable. [...] Ce sera un plaisir de voir ce professeur sévère servir lui-même de jouet aux élèves qu'il a trop souvent châtiés. On lui ôte ses vêtements et on lui attache les mains derrière le dos. La bande d'enfants

51 Corpus Inscriptionum Latinarum, IV 4206 : "Julius est un mignon " ; ibid., IV 4209 : " Helenus, vieux mignon".

52 J.-P. Néraudau, Être enfant à Rome, op. cit., p. 153, qui insiste sur la dimension rituelle des scènes décrites.

53 Suétone, Caligula 27. 
est là, avec ses stylets pointus. Toute la haine que chacun avait accumulée dans sa rancune secrète, il l'épanche avec ardeur, maintenant qu'il peut enfin donner libre cours à sa colère. Les uns lui lancent à la figure et lui brisent sur le visage leurs fragiles tablettes à écrire ; le bois blesse le front et vole en éclats ; le buis enduit de cire craque en frappant les joues sanglantes, le coup écorne la page, la mouille et l'empourpre. Puis d'autres dardent contre lui les piqûres de leurs pointes de fer ; ils se servent de la partie du stylet qui trace sur la cire les sillons de l'écriture, et de celle qui efface les lettres gravées et qui rend à la surface hérissée de signes son poli brillant... (Prudence, Le Livre des couronnes IX, v. 21-54).

Les violences entre étudiants sont connues grâce au témoignage de Libanios. Elles relèvent à la fois de rivalités entre bandes et du bizutage. Dans son Autobiographie (Discours I, 19), le sophiste d'Antioche du IV siècle de notre ère mentionne les affrontements entre étudiants soucieux de " promouvoir le prestige " de leur maître, les " combats que se livrent en pleine Athènes les équipes d'étudiants, les matraques, les couteaux et les pierres et les blessures, et les procès qui s'ensuivent, les plaidoiries et les jugements sur preuve ». Les étudiants arrivés en Attique par les ports du Pirée ou de Sounion sont enlevés pour subir de mauvais traitements (ibid.). Libanios lui-même fut enfermé "dans un tonneau " avant de rencontrer le maître qu'il était venu trouver à Athènes (Discours I, 16). Saint Augustin décrit de même les méfaits de ceux qu'il nomme "les chambardeurs " (euersores), des " effrontés " qui " assaillaient les nouveaux venus intimidés, qu'ils brimaient et outrageaient sans motif, pour repaître leurs joies méchantes " (Confessions III, 3, 6) ${ }^{54}$.

\section{IV - Vers plus de douceur pédagogique?}

\section{1 - Une évolution de la pensée et de la pratique pédagogiques}

Certains Romains de langue latine ou grecque - l'Empire était bilingue - recouraient cependant à des pratiques pédagogiques exemptes de violence. Le poète Horace (65-8 avant J.-C.) mentionnait déjà, sous Auguste, ces maîtres " qui usent de douceur et, plus d'une fois, donnent des friandises aux enfants pour qu'ils consentent à apprendre l'alphabet " (Satires I, v. 25-26).

Au premier siècle de notre ère, Martial, qui évoque l'usage des " fâcheuses férules ", espère que le maître d'école n'aura pas à s'en servir : " Maître d'école, laisse respirer cette naïve jeunesse : puisses-tu en récompense avoir pour auditeurs bien des adolescents aux cheveux bouclés et voir leur essaim, gracieusement groupé autour de ta table, te chérir " (Épigrammes X 62, 1-3). Mais il note que l'intérêt

54 A. D. Booth, "Punishment, Discipline and Riot... ", art. cit., p. 111, parle à leur sujet de gangs. 
des écoliers est fonction de la saison - "En été, si les enfants se portent bien, ils apprennent suffisamment " - et que la férule peut " chômer et dormir jusqu'aux ides d'octobre " (Épigrammes X 62, 11-12).

Plutarque prône une éducation sans coups ni mauvais traitements dans son traité moral De l'éducation des enfants (12) :

Je prétends encore que l'on doit amener les enfants à adopter une bonne conduite en les conseillant et en leur parlant et non, par Zeus, en les frappant et en les maltraitant, car cette dernière méthode semble convenir à des esclaves plutôt qu'à des hommes libres : on y devient comme hébété et horrifié devant les tâches, partie à cause de la douleur, partie aussi à cause des outrages. Louanges et blâmes sont plus utiles pour les personnes libres que n'importe quels sévices [...].

Dans l'Institution oratoire (I, 3, 14-17), Quintilien récuse les punitions corporelles en développant un plaidoyer en six points : elles sont faites pour les esclaves et non pour les enfants libres ; elles relèvent d'un autre âge ; l'enfant va s'endurcir au lieu de s'améliorer ; la présence d'un surveillant assidu près de l'enfant doit les rendre inutiles ; la punition corporelle infligée aux enfants rend difficile une gradation des sanctions vis-à-vis des jeunes gens fautifs ; enfin, la punition peut être contreproductive en incitant l'enfant puni à la violence. L'étude doit être un plaisir : "Il faudra prendre garde qu'incapables encore à cet âge d'aimer les études, les enfants n'en viennent à les détester et n'en redoutent l'amertume qu'ils auront une fois perçue même au-delà des premières années, où ils ne sont pas formés. Que l'étude soit donc un amusement (lusus hic sit), avec des questions et des félicitations, et que l'enfant s'applaudisse toujours de son activité... " (Institution oratoire I, 1, 20).

Dans les Confessions (I, 9), saint Augustin garde un souvenir pénible de son âge d'écolier, tout en reconnaissant qu'il ne travaillait pas assez, se montrant " paresseux à apprendre ". Il dénonce ces " sentiers douloureux où il nous fallait passer ", ces coups reçus des adultes, qu'il assimile à de la torture, ses parents " qui riaient des châtiments " infligés par les maîtres.

Saint Jérôme dresse à la fin du IV ${ }^{\mathrm{e}}$ siècle le tableau idéal de l'éducation d'une jeune chrétienne, où toute violence serait bannie, l'enfant devant être encouragée par un matériel pédagogique attrayant et par des récompenses, dans deux lettres traitant de l'éducation des jeunes Paula et Pacatula.

À propos de Paula :

Qu'on lui fasse des lettres soit de buis, soit d'ivoire, et qu'on les désigne par leur nom. Qu'elle s'en amuse ; qu'ainsi, son amusement même lui soit un enseignement 
[...]. Qu'assembler les syllabes lui vaille une récompense ; qu'on l'y invite encore par les petits cadeaux qui peuvent faire plaisir à cet âge (Lettre 107, 4).

À propos de Pacatula :

D'ici là, qu'elle se contente de savoir les lettres de l'alphabet, d'assembler les syllabes, d'apprendre les noms et de leur associer les verbes ; et, pour l'engager à répéter tout cela de sa voix argentine, qu'on lui donne en récompense un petit gâteau sucré et tout ce qui est agréable au goût ; les fleurs à l'aspect printanier, les gemmes qui rutilent, les poupées qui amusent, elle se dépêchera pour les recevoir [...] ; qu'elle aime ce qu'elle est obligée de dire, de façon que ce ne soit pas une besogne (opus), mais un plaisir (delectatio), non une contrainte (necessitas), mais un libre vouloir (uoluntas) (Lettre 128, 1).

L'attitude de Libanios face à la question des punitions a évolué ${ }^{55}$. Avant 387, il paraît être un partisan convaincu de la méthode traditionnelle : "Tantôt les coups, tantôt les paroles plus cinglantes que le cuir " (Discours LXII, 6). Il s'agit pour lui tant d'inciter au travail que de décourager les étudiants tentés d'aller étudier chez un rival. Il cherchera ensuite à susciter chez ses étudiants le respect plutôt que la crainte, car elle est plus susceptible de mobiliser leur attention et leur intérêt ${ }^{56}$. Il usera cependant encore du fouet pour les petites fautes ${ }^{57}$, pratiquant l'expulsion du cours pour les cas plus graves : "Plus d'une fois, il éleva la voix pour demander qu'on mit la main au collet d'un élève inappliqué et qu'on le jetât dehors " (Discours III, 15).

\section{2 - Une violence persistante}

Ces plaidoyers pour une éducation sans violence ni contrainte sont cependant restés isolés et ils ont été critiqués. Pétrone note dans le Satiricon (IV, 4) que la discipline sévère n'est plus en vigueur à son époque, celle de Néron : "Aujourd'hui, les enfants ne font que jouer à l'école ", ce qu'il condamne : "[Les] études [sont] faites à contre-sens dans le jeune âge ". L'historien Tacite semble faire écho à ces regrets quand il dénonce, dans Le dialogue des orateurs (XXVIII, 2-3), " des maux (mala), nés à Rome ", qui " se sont répandus en Italie et maintenant se propagent dans les provinces ", à savoir "l'oisiveté de la jeunesse, la négligence des parents, l'ignorance des maîtres et l'oubli des mœurs antiques ”.

$55 C f$. André-Jean Festugière, Antioche païenne et chrétienne. Libanius, Chrysostome et les moines de Syrie, Paris, E. de Boccard, 1959, p. 11-113 ; Libanios, Discours, édition par Jean Martin, t. II, Paris, Les Belles Lettres, 1988, p. 254-255.

56 Libanius, Discours II, 20, où il invoque " la douceur (barus) " de son tempérament, LVIII, 1. Cf. Paul Petit, Les étudiants de Libanius, Paris, Nouvelles éditions latines, 1957, p. 148.

Libanios, Lettres 972 (390 de notre ère). 
Dans ses Entretiens (III, 19, 4), le philosophe Épictète s'insurge également contre le relâchement de la discipline éducative traditionnelle en dénonçant le fait qu'un enfant qui avait heurté une pierre par sottise et en "baillant aux corneilles " n'a pas été puni, mais que c'est la pierre qui a été frappée. Les maximes transmises par les documents scolaires d'Égypte grecque et romaine qui érigent le fouet en instrument pédagogique sont attestées jusqu'à la fin de l'Antiquité et au-delà, par exemple dans des documents du VI-VII ${ }^{\mathrm{e}}$ siècle de notre ère où figure leur traduction $\operatorname{copte}^{58}$. Ainsi cette sentence : "Sans coups, personne n'apprend à écrire " ${ }^{59}$.

La question de savoir si la violence scolaire émanant des élèves variait en intensité suivant les niveaux d'éducation est discutée. Il est probable qu'elle se concentrait surtout dans le premier niveau d'éducation et le niveau secondaire, celui des sophistes étant marqué par des relations entre maîtres et étudiants qui étaient moins fondées sur la crainte. Le fait s'expliquerait par l'âge des élèves des deux premiers niveaux, qui sont alors plus turbulents, et peut-être aussi par la mentalité de maîtres plus enclins à l'extrême sévérité60. Il est probable aussi que le recrutement social globalement plus élevé des étudiants incitait les maîtres, seuls responsables de la discipline dans leur classe, à faire attention, car leurs parents étaient mieux armés pour défendre leurs rejetons contre les mauvais traitements. La grand-mère de Kottalos incitait le maître à accabler de coups le malheureux écolier et Libanios se voit injustement reproché par un père d'avoir fouetté son fils ${ }^{61}$. Mais bien des cas d'espèces pouvaient contredire cette vue générale, et la personnalité du maitre devait avoir une grande importance.

\section{3 - Les limites fixées par la loi}

La loi romaine fixait cependant des limites à la violence des maîtres. La punition corporelle est admise dans son principe : "Les maîtres doivent avoir le droit d'infliger une correction modérée (levis castigatio) ", mais " sans dépasser les bor-

58 Papyrus du Vatican publié par Orazio Marucchi, Il museo egiziano Vaticano descritto ed illustrato, Rome, 1899, p. 296 sqq, republié par Dieter Hagedorn et M. Weber, « Die griechisch-koptische Rezension der Menandersentenzen ", Zeitschrift für Papyrologie und Epigraphik, 3 (1968), p. 11-50, en part. p. 28-29 (Folio Ib, 1. 25-26) ; Janine Debut, " Les documents scolaires ", Zeitschrift für Papyrologie und Epigraphik, 63 (1986), p. 251-278, nº 215.

59 Elle est proche d'une sentence de Ménandre : Siegfried Jäkel, Menandri Sententiae, Leipzig, Teubner, 1964, 573 : "Un homme non châtié n'est pas éduqué ".

60 Cf. Raffaella Cribiore, Gymnastics of the Mind..., op. cit., p. 71-72, qui insiste sur le recrutement social des élèves du second niveau et des sophistes ; A. D. Booth, "Punishment, Discipline and Riot... ", art. cit., p. 109, pense en revanche que la violence des maitres était répandue de la même manière dans l'ensemble de la vie scolaire.

61 Cf. P. Petit, Les étudiants de Libanius, op. cit., p. 148. 
nes ${ }^{62}$. À propos de la loi Aquilia, qui date du premier quart du III $^{\mathrm{e}}$ siècle avant J.-C. ${ }^{63}$, le juriste Paul considérait ainsi que " la trop grande sévérité d'un maître est regardée comme une faute de sa part " ${ }^{64}$. Les théoriciens du droit romain donnent quelques exemples de ces abus concernant les esclaves et les apprentis dans un cadre professionnel. Le juriste Julien estime qu'un maître d'école (magister) qui rendrait borgne ou qui tuerait un esclave en le corrigeant devrait être sanctionné, car il a causé un dommage au propriétaire du jeune esclave ${ }^{65}$. Il estime de même qu'un cordonnier qui frapperait son apprenti violemment en lui crevant les yeux devrait réparation à son père. Cette loi, qui s'appliquait très probablement ${ }^{66}$ aux enfants et aux jeunes de statut libre, devait pouvoir être invoquée en cas de blessures causées dans le cadre de l'école romaine.

L'irresponsabilité pénale des jeunes Romains proches de l'âge de 25 ans, âge de la majorité pénale en droit romain, est remise en cause sous l'empereur Julien (331-363). Cette mesure est destinée à briser les violences de jeunes qui s'expriment dans le cadre scolaire, mais aussi (et sans doute surtout) dans le cadre politique ${ }^{67}$.

La présence structurelle de la violence des maîtres est une donnée fondamentale de l'éducation antique. Instrument de la contrainte, la violence des maîtres est aussi destinée à dégager une élite, dans une perspective agonistique qui est au cœur de l'idéal de la civilisation gréco-romaine. Sparte, mais aussi Athènes, en fournissent des illustrations saisissantes. Elle est aussi une réponse - éventuellement préventive - à la violence des enfants, des adolescents et des jeunes gens, tant entre eux qu'envers leurs maîtres. La violence du gymnase, exercée généralement par des citoyens contre de futurs citoyens, est destinée à former à l'obéissance de futurs soldats. Cette violence peut apparaitre dans le cadre scolaire comme une donnée sociale : au mépris envers les maîtres qui peut affecter élèves

62 Digeste IX, 2, 13, 4.

63 Les dates envisagées sont 289, 286 ou une période située entre 289 et 286 avant J.-C.

64 Digeste IX, 2, 6 : Praeceptoris enim nimia saevitia culpae adsignatur.

65 Digeste IX, 2, 5, 3.

66 Ulrich von Lübtow, Untersuchungen zur lex Aquilia de damno iniuria dato, Berlin, Duncker und Humblot, 1971, p. 116-120.

67 Cf. J.-P. Néraudau, Être enfant à Rome, op. cit., p. 222 : la mesure touche les jeunes proches de la puberté, les pubertati proximi : "La loi n’a donc pas découvert chez l'enfant une nouvelle maturité ; elle a simplement dû faire face à des actes de turbulence, de malveillance, à des délits nombreux qui nécessitèrent des mesures de répression nouvelles ". 
et parents, répond la vengeance de maitres emplis de frustrations et d'amertume, hommes libres qui font ce métier par défaut, affranchis et esclaves. Mais les théoriciens comme Platon ou Aristote défendent une conception normative de la violence dans le cadre scolaire, qui n'est remise en cause que de manière marginale par les penseurs et les praticiens sous l'Empire romain. L'usage de la douceur comme pratique pédagogique, dont le théoricien majeur est Quintilien, n’est donc en rien un principe fondateur de l'éducation gréco-romaine. Au total, ces pratiques violentes sont de toute évidence le reflet de la violence diffuse qui affectait la vie des Grecs et des Romains. Le gymnase et l'école dans les mondes grec et romain étaient - ce qui ne saurait surprendre - à la fois un reflet et une propédeutique de la vie sociale dans l'Antiquité gréco-romaine.

Bernard LEGRAS

Université de Reims-Champagne-Ardenne belegras@club-internet.fr 